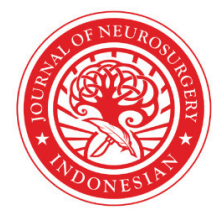

\title{
"Mickey Mouse head aneurysms" - kissing aneurysms of the distal anterior cerebral artery: a case report and literature review
}

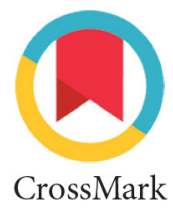

\author{
Sachin Chemate ${ }^{1 *}$, K. Chandrasekar ${ }^{2}$, Shankar Ganesh C. V. ${ }^{2}$, \\ Chetana Govindaraju', M. Balamurugan²
}

ABSTRACT

Background: When two or more adjacent intracranial aneurysms arises from same or different arteries with separate origins and partially adherent walls is referred as "Kissing aneurysm". These are rare aneurysms ${ }^{1}$. Among different locations of kissing aneurysms reported in the literature, kissing aneurysms of bilateral distal anterior cerebral arteries (DACA) - two different aneurysms situated symmetrically on opposite DACA are very rare.

Case Description: 69 years old lady with multiple comorbidities was admitted with anterior inter-hemispheric bleed, Magnetic Resonance Angiography (MRA) and Digital Subtraction Angiography (DSA) showed ruptured bilateral DACA aneurysms in mirror position

to each other forming a Mickey Mouse head appearance. Patient was managed surgically by craniotomy and clipping of both aneurysms.

Conclusion: Kissing aneurysms of bilateral DACA aneurysms are very rare. DSA is essential to be useful for preoperative diagnosis and planning. Basic principle of securing the parent artery proximally and clipping the neck after meticulous dissection should be followed.

Key words: Kissing aneurysm, distal anterior cerebral artery aneurysm, Digital Subtraction Angiography.

Keywords: Kissing aneurysm, distal anterior cerebral artery aneurysm, Digital Subtraction Angiography

Cite This Article: Chemate, S., Chandrasekar, K., C.V.S.G., Govindaraju, C., Balamurugan, M. 2020. "Mickey Mouse head aneurysms" kissing aneurysms of the distal anterior cerebral artery: a case report and literature review. Indonesian Journal of Neurosurgery 3(1): 33-37. D0I: 10.15562/ijn.v3i1.91

${ }^{1}$ Resident, Department of Neurosurgery, Apollo Hospitals, Chennai

${ }^{2}$ Senior Consultant, Department of Neurosurgery, Apollo Hospitals, Chennai

*Corresponding Author: Sachin Chemate; Resident, Department of Neurosurgery, Apollo Hospitals, Chennai sachin.19chemate@gmail.com

Received: 2020-11-11

Accepted: 2020-03-02

Published: 2020-04-01

\section{INTRODUCTION:}

'Kissing' aneurysms were initially defined and described by Jefferson ${ }^{1}$ in 1978. When two or more adjacent Intracranial aneurysms arises from same or different arteries with separate origins and partially adherent walls is referred as "Kissing aneurysm". ${ }^{1}$ They occur in $<1 \%$ of all intracranial aneurysms. ${ }^{2}$ The most common site is the internal carotid artery (ICA) followed by the anterior communicating artery (ACom), distal anterior cerebral artery (ACA), and fenestrated basilar artery (BA)..$^{2-8}$ Kissing aneurysms of bilateral distal anterior cerebral arteries (DACA) are rare and till date only 12 cases reports have been reported. We report one such case which was managed surgically by craniotomy and clipping of both aneurysms.

\section{CASE REPORT}

69 years old lady was admitted to emergency department with complaints of one episode of sudden loss of consciousness lasting for 5-10 minutes, followed by headache. She was known case of type II diabetes mellitus since 15 years and systemic hypertension since 10 years on regular management and previous history of well differentiated squamous cell carcinoma of oesophagus diagnosed 5 years ago for which she underwent radiation and achieved remission. On examination, the patient was E4V4M6, vitals were stable, pupil was normal sized and reacting to light, and there were no focal neurological deficits. Due to previous history of carcinoma oesophagus and patient's history, cerebral metastasis was suspected and MRI brain was done but surprisingly it showed anterior interhemispheric bleed with corpus callosum hematoma (Figure 1a and $1 \mathrm{~b}$ ) and Magnetic resonance angiography (MRA) of the brain was suggestive of two adjacent aneurysm on bilateral distal anterior cerebral artery (ACA) at the junction of pericallosal and callosomarginal arteries (Figure 2a and d). Digital subtraction angiography (DSA) was done which showed two adjacent aneurysms over each DACA at the junction of pericallosal and callosomarginal arteries. Right side aneurysm was size $9.4 \times 8.7 \mathrm{~mm}$ with a neck measuring $2.5 \mathrm{~mm}$ with a daughter lobule and superiorly projecting rupture site. Left-sided aneurysm measured size $7.6 \times 5.5 \mathrm{~mm}$ with a neck of $2.5 \mathrm{~mm}$. Both were directed antero-superiorly. Both A1 ACAs were of the same size with good 

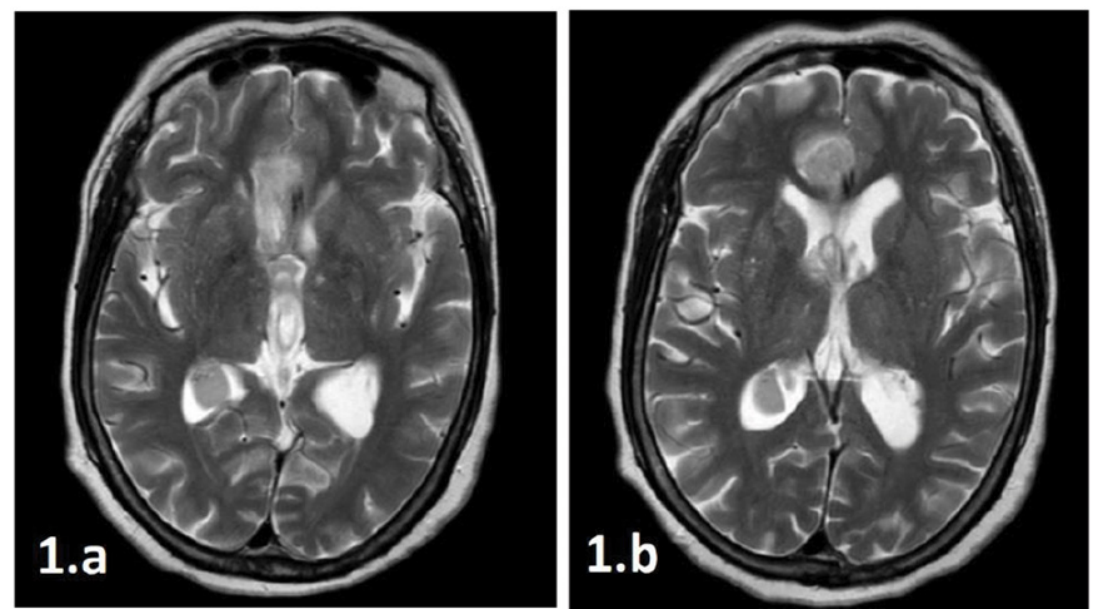

Figure $1 \mathbf{a}$ and $\mathbf{1 b}$. T2W MRI brain showing anterior interhemispheric bleed
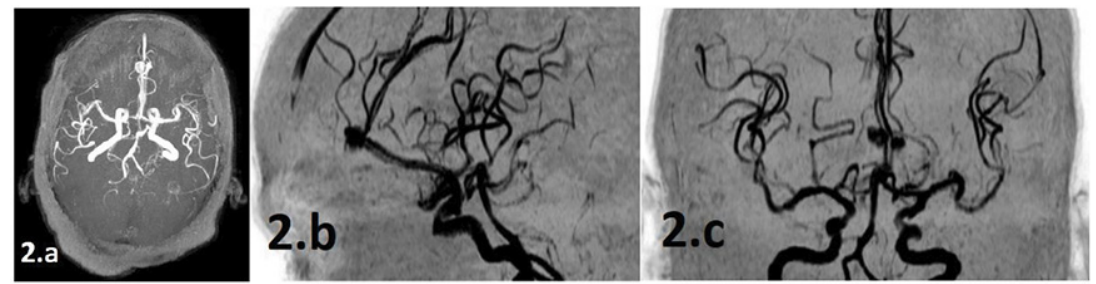

Figure $\mathbf{2 a}, \mathbf{2} \mathbf{b}$ and $\mathbf{2 c}$. MRA showing of two adjacent aneurysms over each DACA
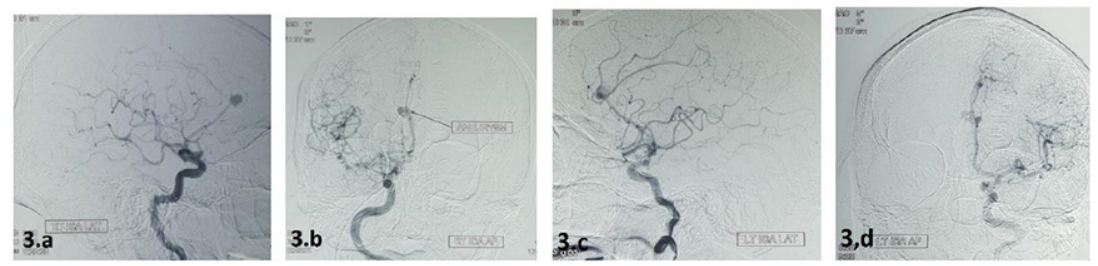

Figure $3 a, 3 b, 3 c$ and $3 d$ : DSA showing of two adjacent aneurysms over each DACA

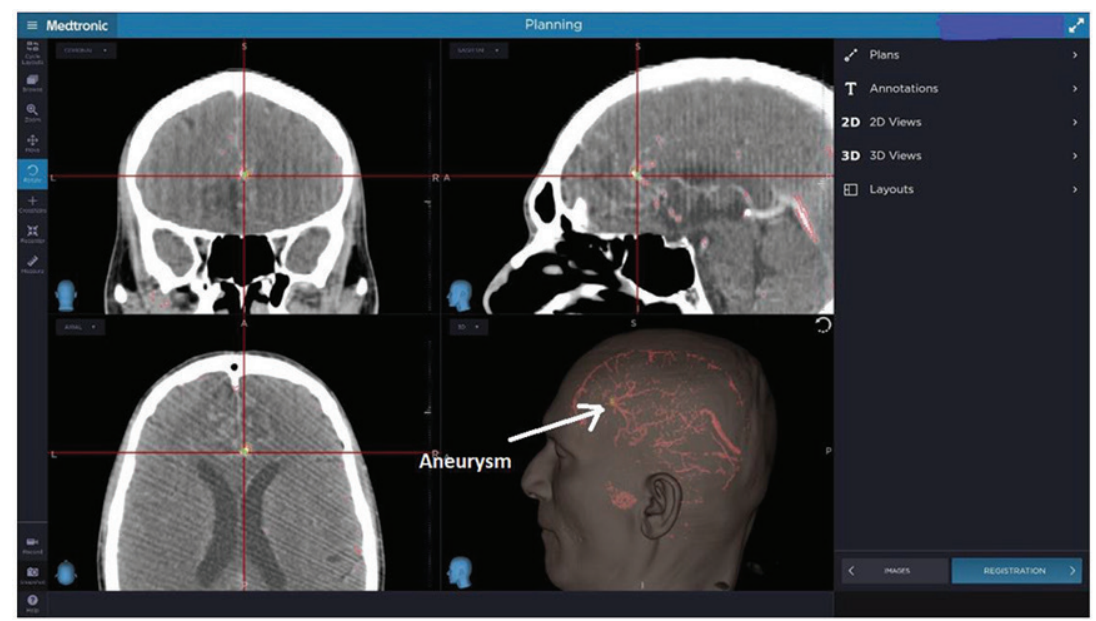

Figure 4. Navigation system showing aneurysm (green dot) pointed by white arrow. crossflow. Focal areas of segmental spasm were noted in right pericallosal arteries (Figure 3a-d).

The patient underwent surgery; right frontal craniotomy was done. Anterior interhemispheric approach was utilized. With help of navigation (Figure 4), pericallosal and callosomarginal arteries of the left side was identified and traced proximally. Right DACA aneurysm arising from the junction of pericallosal and callosomarginal artery directing anterosuperiorly was visualized (Figure $5 \mathrm{a}$ and $5 \mathrm{~b}$ ). Temporary proximal clip was applied over right A2 and right DACA aneurysm was clipped. Left DACA aneurysm was identified, it was buried in the brain parenchyma with overlying hematoma and adhesions. Proximal control over right A2 achieved with temporary clip. While dissecting dome of aneurysm there was intraoperative rupture, which was managed with bipolar cautery and permanent clip applied at the neck of right DACA aneurysm. Intraoperative Indocynine Green (ICG) dye showed patent proximal and distal DACA, both the aneurysms were completely secured and there was no residual neck (Figure 5c).

Minimal brain retraction was used. Postoperatively, patient was extubated immediately. Postoperatively patient had no new deficit. As our institutional protocol, patient was managed with triple-H therapy. CT brain was done on postoperative day 1 , which showed no evidence of infarct or haemorrhage (Figure $6 \mathrm{a}$ and $6 \mathrm{~b}$ ). Patient was discharged on postoperative day day 7 without any neurological deficit.

\section{DISCUSSION:}

\section{Epidemiology}

DACA aneurysms have been reported to have an average incidence of $4.4 \% .^{14}$ Bilateral DACA "mirror image aneurysms" are extremely rare $^{9-21}$, with majority of reports from Japanese literature (Table 1).

\section{Pathophysiology}

Laitenen and Snellman ${ }^{22}$ postulated that a supreme anterior communicating artery, a bridging artery located at the bifurcation of A2 into the pericallosal and callosomarginal arteries and other embryological connections like Azygos ACA, and triple ACAs might represent an embryological remnant and lead to flow disturbance in anterior cerebral artery bifurctation causing bilateral, symmetrical aneurysms. However, no vascular anomaly was detected in our case. Yasargil and Carter ${ }^{23,24}$ treated 13 patients with DACA aneurysms with microsurgical techniques and found that in there were two cases where the aneurysmal sacs were connected. Based on this they suggested that, some 
embryological variation, such as a supreme ACoA, may cause a flow disturbance leading to aneurysm formation. Jefferson ${ }^{1}$ suggested hereditary basis for pathogenesis of bilateral DACA aneurysm but still remains unclear. Wanifuchi et $\mathrm{al}^{25}$ described female dominance of bilateral DACA aneurysm but other multiple aneurysms also has higher frequency in females.

\section{DIAGNOSIS}

CT angiography, MRA, and DSA are most useful
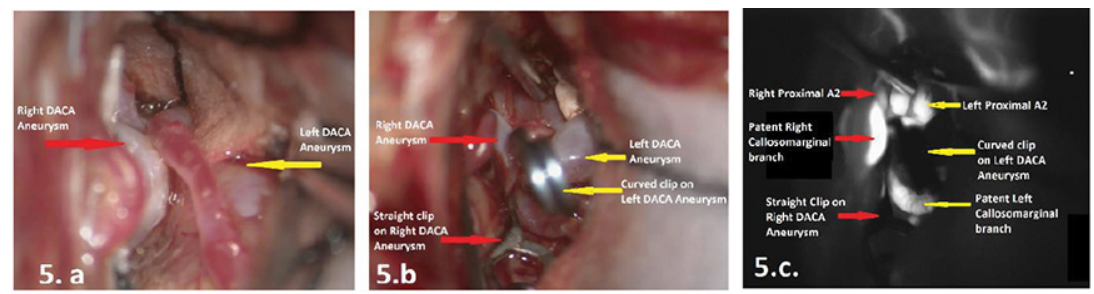

Figure 5.a and 5.b. intraoperative images showing mirror DACA aneurysms
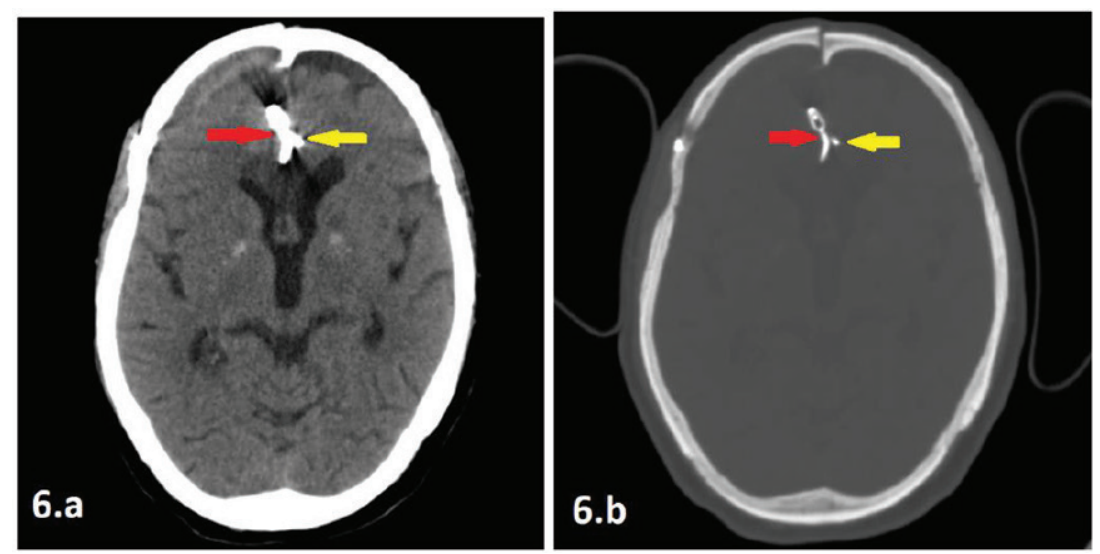

Figure $\mathbf{6 a}$ and $\mathbf{6 b}$. CT brain showing clips applied on both DACA aneurysm for the diagnosis of bilateral DACA aneurysms. Although MRA is very sensitive and specific for distal aneurysm and 3D-reconstructions are also available in this imaging, however, DSA provides complete study of intracranial vessels in real time. Vasospasm can also be diagnosed during angiogram in early phases. Other distal Incidental aneurysms can be seen in angiograms. It also gives very critical information regarding aneurysm location, shape, size of neck, branch vessel, and cross circulation; which helps surgeon to decide whether to go for clipping or coiling.

\section{Management}

The most definite management at the bilateral DACA aneurysm is clipping the aneurysm. Bilateral DACA aneurysm has tendency to rupture so it presents with subarachnoid haemorrhage and anterior interhemispheric hematoma. Incidence of rebleed is also very high in DACA aneurysm. Through anterior interhemispheric approach we can not only achieve a proximal control on A2 and secure the aneurysm but also it provides an access evacuation of for hematoma and mass effect and thorough washing of cranial cavity with normal saline during surgery also removes blood by products which decreases the intensity and duration of vasospasm. Aneurysm occlusion rate is very high with clipping and rebleeding or recurrence is very rare after clipping. Challenges of craniotomy and clipping are narrow working corridor, edematous brain, adhesions and buried dome of aneurysm in the surrounding brain parenchyma. Dome of the aneurysms is always directed towards the surgeon and proximal A2 is inferior to aneurysm when visualised from working corridor, hence proximal

Table 1.

\begin{tabular}{|c|c|c|c|}
\hline No. & Year & Auther & Case reports \\
\hline 1 & 1988 & Megele et al $^{9}$ & A case of symmetrical pericallosal aneurysms with recurrent haemorrhage \\
\hline 2 & 1989 & Niijima et $\mathrm{al}^{10}$ & Bilateral pericallosal artery aneurysms in a mirror position \\
\hline 3 & 1995 & Mori et $\mathrm{al}^{11}$ & Kissing aneurysms of DACA demonstrated by magnetic resonance angiography \\
\hline 4 & 2001 & Moon et $\mathrm{al}^{12}$ & Kissing aneurysms of DACA distal anterior cerebral arteries \\
\hline 5 & 2002 & Sousa et $\mathrm{al}^{13}$ & $\begin{array}{l}\text { Mirror image DACA aneurysms. A case report of two patients with review of } \\
\text { literature }\end{array}$ \\
\hline 6 & 2003 & Kawamata et $\mathrm{al}^{14}$ & $\begin{array}{l}\text { Bilateral DACA aneurysms associated with supreme anterior cerebral artery: case } \\
\text { report }\end{array}$ \\
\hline 7 & 2006 & Ahn et $\mathrm{al}^{15}$ & Kissing aneurysms of DACA \\
\hline 8 & 2006 & Dinc et $\mathrm{al}^{16}$ & DACA mirror aneurysms and middle cerebral artery aneurysms \\
\hline 9 & 2010 & Meysam Alimohammadi et $\mathrm{al}^{17}$ & Bilateral "Kissing" Aneurysms of the Distal Pericallosal Arteries \\
\hline 10 & 2011 & Choi CY at al ${ }^{18}$ & Kissing aneurysms of the distal anterior cerebral artery \\
\hline 11 & 2013 & E. Enesi at all ${ }^{19}$ & $\begin{array}{l}\text { Mirror Image Distal Anterior Cerebral Artery Aneurysms Treated with Coil } \\
\text { Embolization }\end{array}$ \\
\hline 12 & 2018 & Chuan-Yi Fu et al ${ }^{20}$ & Kissing aneurysms of the distal anterior cerebral artery. \\
\hline 13 & 2018 & Singh SK ${ }^{21}$ & Mirror image of bilateral DACA aneurysm with its successful surgical management. \\
\hline
\end{tabular}


control is challenge and chance of intraoperative rupture is high. Only one case report is available in literature about the experience of coiling.

Our patient was taken up for clipping after angiogram. During the operative procedure, retraction was applied first on the right medial frontal lobe. Parent artery and dome of left aneurysm was identified. Dome was followed inferiorly up to the neck and the clip was applied on proximal A2. After securing left-sided aneurysm completely, right-sided A2 proximal clip was applied. Dome and neck of the right DACA aneurysm was then dissected and clip applied. However while dissecting the aneurysm aneurysm ruptured in spite of temporary clip on proximal A2. Recurrent use of temporary clips loses there occlusion force resulting in only partial occlusion. Intraoperative rupture was managed with bipolar cautery over dome and multiple clipping over the bleb. After securing the bleb rupture, final clip was applied at the neck.

\section{CONCLUSION}

DSA is essential for diagnosis of multiple intracranial aneurysm. Mirror images of bilateral DACA aneurysms are very rare. They are specific type of aneurysms with probably derived from embryological rearmament. Basic surgical strategy of securing the parent artery and clipping the neck after meticulous dissection of each aneurysm separately is key for successful clipping of aneurysm, as chance of intraoperative rupture is very high.

\section{CONFLICT OF INTEREST}

There are no conflicts of interest.

\section{FINANCIAL SUPPORT AND SPONSOR- SHIP}

There is no funding available for this case report.

\section{AUTHORS CONTRIBUTIONS}

All authors equally contributed immensely.

\section{ABBREVIATION}

DACA - Distal Anterior Cerebral Arteries MRA - Magnetic Resonance Angiography DSA - Digital Subtraction Angiography ACom - Anterior Communicating Artery ACA - Anterior Cerebral Artery

BA - Basilar Artery

Fig - Figure

ICG - Intraoperative Indocynine Green CT - Computed Tomography

\section{REFERENCE}

1. Jefferson A: The significance for diagnosis and for surgical technique of multiple aneurysms of the same internal carotid artery. Acta Neurochir (Wien) 41: 23-37, 1978.

2. Choi CY, Han SR, Yee GT and Lee CH: Kissing aneurysms of the distal anterior cerebral artery. J Clin Neurosci 18 : 260-262, 2011

3. Komiyama M, Yasui T, Tamura K, et al. "Kissing aneurysms" of the internal carotid artery. Neurol Med Chir (Tokyo) 1994;34:360-4.

4. Yasargil MG. Internal carotid artery aneurysms. In: Yasargil MG, editor. Microneurosurgery, vol. 2. New York: Thieme Stratton; 1984. p. 33-123.

5. Wanifuchi $\mathrm{H}$, Shimizu $\mathrm{T}$, Higa $\mathrm{T}$, et al. Kissing mirror image anterior communicating artery aneurysms-case report. Neurol Med Chir (Tokyo) 2001;41:29-32.

6. Imai K. Kissing aneurysms of the internal carotid artery: case report. Jpn J Neurosurg (Tokyo) 2001;10:801-6.

7. Date I, Ogihara K, Tamiya T, et al. "Kissing" bilateral large carotid-ophthalmic aneurysms. A case report. Neurosurg Rev 1998; 21:281-3.

8. Ide M, Hagiwara S, Tanaka N, et al. Bilateral ophthalmic segment "kissing" aneurysms presenting with subarachnoid hemorrhage-case report. Neurol Med Chir (Tokyo) 2002; 42:427-30.

9. Megele R, Gruss P, Lehr H. A case of symmetrical pericallosal aneurysms with recurrent hemorrhage. Neurochirurgia (Stuttg). 1988;31:154-156. [Article in German]

10. Niijima KH, Yonekawa Y, Kawano T. Bilateral pericallosal artery aneurysms in a mirror position. No Shinkei Geka. 1989;17:779-781. [Japanese].

11. Mori T, Fujimoto M, Shimada K, et al. Kissing aneurysms of distal anterior cerebral arteries demonstrated by magnetic resonance angiography. Surg Neurol 1995; 43:497-9.

12. Moon SJ, Kim TS, Lee JH, et al. Kissing aneurysms of distal anterior cerebral arteries: a case report. Korean J Cerebrovasc Dis. 2001;3:70-72. [Korean].

13. Sousa J, Iyer V, Roberts G. "Mirror image" distal anterior cerebral artery aneurysms. A case report of two patients with review of literature. Acta Neurochir (Wien). 2002;144:933-935.

14. Kawashima M, Matsushima T, Sasaki T. Surgical strategy for distal anterior cerebral artery aneurysms: microsurgical anatomy. J Neurosurg. 2003;99:517-525.

15. Ahn H-J, Koh H-S, Kim Y. Kissing aneurysms of distal anterior cerebral arteries. J Korean Neurosurg Soc. 2006:39:238-240

16. Dinc C, Iplikcioglu AC, Bikmaz K, et al. Distal anterior cerebral artery mirror aneurysms and middle cerebral artery aneurysms. Neurol Med Chir (Tokyo). 2006;46:438440.

17. Meysam Alimohammadi, Mohammad Shirani Bidabadi, Abbas Amirjamshidi, Bilateral "Kissing" Aneurysms of the Distal Pericallosal Arteries Report of a Case and Review of the Literature. Neurosurg Q, 2010; 20:4.

18. Choi CY, Han SR, Yee GT and Lee CH: Kissing aneurysms of the distal anterior cerebral artery. J Clin Neurosci. 2011; 18: 260-262.

19. E. Enesi, A. Rroji, M. Demneri, G. Vreto, M. Petrela. Mirror Image Distal Anterior Cerebral Artery Aneurysms Treated with Coil Embolization A Report of Two Cases and Literature Review. Interventional Neuroradiology. 2013; 19: 49-55.

20. Chuan-Yi Fu, Jian-Long Chen at al. Kissing aneurysms of the distal anterior cerebral artery, a case report and literature review. Experimental and Therapeutic Medicine. 2018; 15: 3471-3476. 
21. Singh SK, Jain K, Jain VK, Saroha A. Mirror image of bilateral DACA aneurysm with its successful surgical management. Surg Neurol Int 2018;9:80.

22. Laitenen L, Snellman A. Aneurysms of the pericallosal artery: a study of 14 cases verified angiographically and treatment mainly by direct surgical attack. J Neurosurg 1960;17:447-58.

23. Yasargil MG, Carter LP. Saccular aneurysms of the distal anterior cerebral artery. J Neurosurg. 1974; 39: 218-223.
24. Yasargil MG. Surgery of the intracranial aneurysms and results. In Yasargil MG ed. Microsurgery, Vol II, Clinical considerations. Stuttgart: Georg Theme Verlag; 1984. p. 224-231.

25. Wanifuchi H, Shimizu T, Higa T, et al. Kissing mirror image anterior communicating artery aneurysms-case report. Neurol Med Chir (Tokyo) 2001;41:29-32.

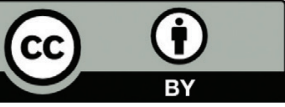

This work is licensed under a Creative Commons Attribution 